\title{
Correction to: MiR-96 promotes apoptosis of nucleus pulpous cells by targeting FRS2
}

\author{
Xiaoxia Yang ${ }^{1} \cdot$ Hengping Liu ${ }^{1} \cdot$ Qingfeng Zhang ${ }^{1} \cdot$ Kan Liu $^{1} \cdot$ Dong Yu $^{1} \cdot$ Yi Zhang $^{1} \cdot$ Zongting Shi $^{1}$
}

Published online: 8 September 2020

(c) Japan Human Cell Society 2020

Correction to: Human Cell

https://doi.org/10.1007/s13577-020-00389-9

In the original publication of the article, the "Funding" and "Ethical approval" were incorrectly published. The corrected information is given below.

Funding This study was funded by Beijing university of Chinese medicine 2018 university-level project (young teachers project) (2018-JYBZZ-JS193).

Ethical approval All of the experiments were approved by the Ethics Committees of the Beijing university of Chinese Medicine Third Affiliated Hospital. The informed consent forms were signed by patients or their families.

The original article can be found online at https://doi.org/10.1007/ s13577-020-00389-9.

Xiaoxia Yang

yangxiaoxia_12@126.com

1 Department of Spine, Beijing University of Chinese

Medicine Third Affiliated Hospital, No.51 Xiaoguan Street,

Outside, Andingmen, Beijing 100029, China 